\title{
Relations Between Quantum Maps and Quantum States
}

\author{
M. Asorey \\ Departamento de Física Teórica, Universidad de Zaragoza \\ 50009 Zaragoza, Spain \\ e-mail: asorey@saturno.unizar.es \\ A. Kossakowski \\ Institute of Physics, Nicolaus Copernicus University \\ Toruń $87-100$, Poland \\ e-mail: kossak@phys.uni.torun.pl \\ G. Marmo \\ Dipartimento di Scienze Fisiche, Universitá Federico II di Napoli \\ and \\ INFN, Sezione di Napoli \\ Complesso Univ. di Monte Sant'Angelo, Via Cintia, 80125 Napoli, Italy \\ e-mail: marmo@na.infn.it \\ E. C. G. Sudarshan \\ Department of Physics, University of Texas at Austin \\ Austin, Texas 78712-1081 \\ e-mail: sudarshan@physics.utexas.edu
}

August 26, 2018

\begin{abstract}
The relation between completely positive maps and compound states is investigated in terms of the notion of quantum conditional probability.
\end{abstract}

\section{Introduction}

In quantum information two problems play a relevant role, the first one concerns the study of the dynamical change of states of a system by means of completely positive maps, commonly called channels, the second one is to describe corre- 
lations between the initial and final states; such correlations are described by compound states.

The connection between the two concepts is based on a very general principle. Indeed, in any Hilbert space $\mathcal{H}$ there is an one-to-one correspondence between the set $\mathcal{P}_{q}^{p}$ of $p$-contravariant $q$-covariant tensors and the set $\mathcal{P}_{p+q}$ of $p+q$ covariant tensors. The equivalence is due to the identification of $\mathcal{H}$ and its dual space $\mathcal{H}^{*}$ by means of the hermitian product. Consequently, endomorphisms of $\mathcal{H}$ which are in $\mathcal{P}_{1}^{1}$ are in one-to-one correspondence with 2-covariant tensors in $\mathcal{P}_{2}$. In particular, if we consider the Hilbert spaces of Hilbert-Schmidt operators on $\mathcal{H}_{1}$ and $\mathcal{H}_{2}$, any map of the Hilbert-Schmidt operators on $\mathcal{H}_{1}$ into those on $\mathcal{H}_{2}$ is associated to a state on the tensor product of the spaces of Hilbert-Schmidt operators on $\mathcal{H}_{1}$ and $\mathcal{H}_{2}$.

The correspondence can also be formulated at $C^{*}$-algebraic level. However, only the finite-dimensional case will be considered here.

Let us consider two systems described by $\left(M_{n}, S\left(M_{n}\right)\right)$ and $\left(M_{m}, S\left(M_{m}\right)\right)$. The first one describes an initial (input) system and the second one a final (output) system. The symbol $M_{n}$ stands for the algebra of $n \times n$ complex matrices and the symbol $S\left(M_{n}\right)$ stands for the set of all states on $M_{n}$, i.e. the set of all density matrices. Moreover, $I_{n}$ denotes the identity matrix in $M_{n}$. Let us consider a map $\varphi^{*}: S\left(M_{n}\right) \rightarrow S\left(M_{m}\right)$, such that its dual map $\varphi: M_{m} \rightarrow M_{n}$ is completely positive and normalized, i.e. $\varphi\left(I_{m}\right)=I_{n}$.

For an initial state $\rho \in M_{n}$ and final state $\varphi^{*}(\rho) \in M_{m}$, a composite state $\omega \in S\left(M_{n} \otimes M_{m}\right)$ should satisfy the following two conditions:

i) $\quad \omega\left(a \otimes I_{m}\right)=\rho(a)$, for all $a \in M_{n}$

ii) $\quad \omega\left(I_{n} \otimes b\right)=\varphi^{*}(\rho)(b)$ for all $b \in M_{m}$.

It is well known that joint probability measures do not generally exist for quantum systems, therefore it is difficult to define a compound state $\omega$ satisfying the above conditions.

The first construction of a compound state $\omega$ satisfying the above two conditions has been given by Ohya $[1,2]$. Let $\rho \in M_{n}$, then $\rho$ has the following spectral decomposition

$$
\rho=\sum_{k} \lambda_{k} m_{k} \rho_{k},
$$

where

$$
\rho_{k}=\frac{1}{m_{k}} p_{k}, \quad m_{k}=\operatorname{Tr} p_{k},
$$

$\lambda_{k}$ are the eigenvalues of $\rho$, and $p_{k}$ are eigenprojectors of $\rho$, respectively.

Then for any $\varphi^{*}: S\left(M_{n}\right) \rightarrow S\left(M_{m}\right)$ the compound state $\omega_{\varphi} \in S\left(M_{n} \otimes M_{m}\right)$ has the form

$$
\omega_{\varphi}=\sum_{k} \lambda_{k} m_{k} \rho_{k} \otimes \varphi^{*}\left(\rho_{k}\right) .
$$

Let us observe that $\omega_{\varphi}$ is a separable state on $M_{n} \otimes M_{m}, \varphi$ is a positive normalized map $\varphi: M_{m} \rightarrow M_{n}$, and the construction of $\omega_{\varphi}$ is non-linear with respect to $\rho$. 
We notice that the Ohya compound state can be constructed in the case of general $C^{*}$-algebraic setting.

The construction of compound states which will be studied in the present paper can be described as follows.

Let $\sigma \in S\left(M_{n} \otimes M_{m}\right)$, and suppose that

$$
\operatorname{tr}_{\mathbb{C}^{m}} \sigma=\sigma_{1}>0
$$

Then, one can define the following operator

$$
\begin{gathered}
\pi(\sigma): \mathbb{C}^{n} \otimes \mathbb{C}^{m} \longrightarrow \mathbb{C}^{n} \otimes \mathbb{C}^{m} \\
\pi(\sigma)=\left(\sigma_{1}^{-1 / 2} \otimes I_{m}\right) \sigma\left(\sigma_{1}^{-1 / 2} \otimes I_{m}\right),
\end{gathered}
$$

which has the properties

$$
\begin{gathered}
\pi(\sigma) \geq 0 \\
\operatorname{tr}_{\mathbb{C}^{m}} \pi(\sigma)=I_{m} .
\end{gathered}
$$

It follows from (1.4) and (1.5) that the operator $\pi$ is the quantum analogue of classical conditional probability. Another definition of quantum conditional probability has been given in [3].

Definition 1 A map

$$
\pi: \mathbb{C}^{n} \otimes \mathbb{C}^{m} \longrightarrow \mathbb{C}^{n} \otimes \mathbb{C}^{m}
$$

is a quantum conditional probability (QCPO) iff satisfies (1.6) and (1.7).

For a given $\pi$ and any $\rho \in S\left(M_{n}\right)$ one can define a compound state

$$
\omega=\left(\rho^{1 / 2} \otimes I_{m}\right) \pi\left(\rho^{1 / 2} \otimes I_{m}\right),
$$

which has the following properties

$$
\operatorname{tr}_{\mathbb{C}^{m}} \omega=\rho
$$

and

$$
\operatorname{tr}_{\mathbb{C}^{n}} \omega=\operatorname{tr}_{\mathbb{C}^{n}} \pi\left(\rho \otimes I_{m}\right)=\varphi^{*}(\rho),
$$

where

$$
\varphi^{*}: S\left(M_{n}\right) \longrightarrow S\left(M_{m}\right) .
$$

The study of the relation between $\varphi^{*}$ and $\pi$ is based on duality between quantum maps and composite states which has been investigated in detail in $[4,11]$ (see also references therein). 


\section{Classification of Composite States and Posi- tive Maps}

It has been shown above that the construction of composite states is based on the notion of quantum conditional probability operator $(Q C P O) \pi: \mathbb{C}^{n} \otimes \mathbb{C}^{m} \rightarrow$ $\mathbb{C}^{n} \otimes \mathbb{C}^{m}$. In what follows the case $m=n$ will be considered for simplicity.

Let $\mathcal{H}$ be a Hilbert space and $\mathcal{H}_{1}=\mathcal{H}_{2}=\mathcal{H}$, the following order in the tensor product $\mathcal{H} \otimes \mathcal{H}=\mathcal{H}_{2} \otimes \mathcal{H}_{1}$ will be used, and the partial trace with respect to the Hilbert space $\mathcal{H}_{a}$ will be denoted by $\operatorname{tr}_{a}$.

Let $\left\{e_{1}, \ldots, e_{n}\right\}$ be a fixed orthonormal basis in $\mathbb{C}^{n}$ and $e_{k l}=e_{k}\left(e_{l}, \cdot\right)$ be the corresponding basis in $M_{n}$, then $a \in M_{n}$ can be written in the form

$$
a=\sum_{i, j=1}^{n}\left(e_{i}, a e_{j}\right) e_{i j}=\sum_{i, j=1}^{n} \operatorname{tr}\left(a e_{i j}^{*}\right) e_{i j},
$$

and the transpose map $\mathcal{T}: M_{n} \rightarrow M_{n}$ (with respect to the basis $\left\{e_{1}, \ldots, e_{n}\right\}$ ) has the form

$$
\mathcal{T}(a)=\sum_{i, j=1}^{n}\left(e_{j}, a e_{i}\right) e_{i j}=\sum_{i, j=1}^{n} e_{i j} a e_{i j} .
$$

A generic element $x \in \mathbb{C}^{n} \otimes \mathbb{C}^{n}$ can be written in the form

$$
x=\sum_{i=1}^{n} x_{i} \otimes e_{i}=\sum_{i=1}^{n}\left(a e_{i}\right) \otimes e_{i},
$$

where $x_{1}, x_{2}, \ldots, x_{n} \in \mathbb{C}^{n}$ and $a \in M_{n}$.

With every $a \in M_{n}$, such that $\operatorname{tr}\left(a^{*} a\right)=1$, one can associate one-dimensional projections $p_{a}: \mathbb{C}^{n} \otimes \mathbb{C}^{n} \rightarrow \mathbb{C}^{n} \otimes \mathbb{C}^{n}$

$$
p_{a}=\sum_{i, j=1}^{n} a e_{i j} a^{*} \otimes e_{i j} .
$$

Moreover, two projections $p_{a}$ and $p_{b}$ are orthogonal provided that $\operatorname{tr}\left(a^{*} b\right)=0$. As a consequence of $(2.4)$ any positive operator $\hat{A}: \mathbb{C}^{n} \otimes \mathbb{C}^{n} \rightarrow \mathbb{C}^{n} \otimes \mathbb{C}^{n}$ has the form

$$
\widehat{A}=\sum_{i, j=1}^{n} \sigma_{i j} \otimes e_{i j}=\sum_{i, j=1}^{n} \varphi\left(e_{i j}\right) \otimes e_{i j},
$$

where

$$
\varphi\left(e_{i j}\right)=\sum_{\alpha=1}^{n^{2}} \lambda_{\alpha} a_{\alpha} e_{i j} a_{\alpha}^{*}
$$

and

$$
\lambda_{\alpha} \geq 0, \quad \operatorname{tr}\left(a_{\alpha} a_{\beta}^{*}\right)=\delta_{\alpha \beta},
$$

i.e. $\left\{a_{1}, a_{2}, \ldots, a_{n^{2}}\right\}$ is an orthonormal basis in $M_{n}$. 
Relation (2.5) can also be rewritten in the form

$$
\hat{\sigma}=(\varphi \otimes \mathrm{id}) \sum_{i, j=1}^{n} e_{i j} \otimes e_{i j}
$$

which gives the relation between elements of $\left(M_{n} \otimes M_{n}\right)^{+}$and completely positive maps in $M_{n}$. In order to classify the states on $M_{n} \otimes M_{n}$ it is convenient to introduce the following cones in $M_{n} \otimes M_{n}$ :

$$
V_{s}=\operatorname{conv}\left\{\sum_{i, j=1}^{n} a e_{i j} a^{*} \otimes e_{i j}: a \in M_{n}, \operatorname{rank} a \leq s\right\}
$$

where conv $X$ means convex (not normalized) set generated by elements of $X$, and

$$
V^{s}=(\mathrm{id} \otimes \mathcal{T}) V_{s}
$$

where $\mathcal{T}$ is the transpose map on $M_{n}$, i.e.

$$
V^{s}=\operatorname{conv}\left\{\sum_{i, j}^{n} a e_{i j} a^{*} \otimes e_{j i}: a \in M_{n}, \operatorname{rank} a \leq s\right\} .
$$

It follows from (2.8) and (2.9) that the following chains of inclusions

$$
\begin{gathered}
V_{1} \subset V_{2} \subset \ldots \subset V_{n}, \quad V^{1} \subset V^{2} \subset \ldots \subset V^{n}, \\
V_{1} \cap V^{1} \subset V_{2} \cap V^{2} \subset \ldots \subset V_{n} \cap V^{n}
\end{gathered}
$$

hold true.

It is clear that $V_{n}$ coincides with the cone $\left(M_{n} \otimes M_{n}\right)^{+}$of all positive semidefinite elements of $M_{n} \otimes M_{n}, V_{1}=V^{1}$ is the cone generated by elements $a \otimes b$, where $a, b$ are positive elements of $M_{n}$, i.e. $V_{1}$ coincides with separable (not normalized) states on $M_{n} \otimes M_{n}$, while $V_{n} \cap V^{n}$ is the set of all (not normalized) PPT states on $M_{n} \otimes M_{n}$ (by definition).

Using the results of $[4,5,6]$ the above cones can be used to classify positive maps. Let $P_{s}, P^{s}$ and $P_{s} \cup P^{r}$ be the cones of $s$-positive, $s$-copositive maps and sums of $s$-positive and $r$-copositive ones, respectively. One can verify that

$$
\begin{aligned}
\varphi \in P_{s} & \Longleftrightarrow(\varphi \otimes \mathrm{id}) V_{s} \in\left(M_{n} \otimes M_{n}\right)^{+} \\
\varphi \in P^{s} & \Longleftrightarrow(\varphi \otimes \mathrm{id}) V^{s} \in\left(M_{n} \otimes M_{n}\right)^{+}
\end{aligned}
$$

and

$$
\varphi \in P_{s} \cup P^{r} \Longleftrightarrow(\varphi \otimes \mathrm{id}) V_{s} \cap V^{r} \in\left(M_{n} \otimes M_{n}\right)^{+} .
$$

Relations (2.12) can be considered as an extension of the Horodecki theorem [7] which gives the characterization of the cone $V_{1}$ in terms of positive maps. It should be pointed out that our knowledge concerning the above mentioned cones is rather poor. In fact only the structure of cones $V_{n}$ and $V^{n}$ is known.

In the $n$-dimensional case an example of an element $V_{2} \cap V^{2}$ which is not separable has been given in [8].

The cones $V_{r}$ and $V^{r}$ and $V_{r} \cap V^{s}$ can be used for classification of completely positive maps. 
Definition 2 A completely positive map $\varphi: M_{n} \longrightarrow M_{n}$ is said to be $s$-completely positive if

$$
\sum_{i, j=1}^{n} \varphi\left(e_{i j}\right) \otimes e_{i j} \in V_{s}
$$

and $(r, s)$-completely positive if

$$
\sum_{i, j=1}^{n} \varphi\left(e_{i j}\right) \otimes e_{i j} \in V_{r} \otimes V^{s}
$$

Let us observe that the set $\mathcal{P}_{r s}$ of all $(r, s)$-completely positive maps is a subset of $P_{n} \cap P^{n}$, the subset of maps which are completely positive and completely copositive. On the other hand, $(r, s)$-completely positive maps generate PPTstates since the inclusion $V_{r} \cap V^{s} \subseteq V_{n} \cap V^{n}$ holds.

It is also convenient to consider $s$-completely positive maps which are $k$ copositive, i.e. elements of the set $P_{n} \cap P^{k}(k<n)$ which generate NPT states. The construction of composite states in terms of QCPO will be used to find out some classes of PPT and NPT states.

Indeed, taking into account (2.5), one finds out that the general form of $\pi$ is given by

$$
\pi=\sum_{i, j}^{n} \varphi\left(e_{i j}\right) \otimes e_{i j},
$$

where $\varphi$ is a completely positive normalized map in $M_{n}$, i.e., $\varphi\left(I_{n}\right)=I_{n}$. It follows from (2.14) and (1.8) that the composite state can be written in the form

$$
\omega=\sum_{i, j=1}^{n} \rho^{1 / 2} \varphi\left(e_{i j}\right) \rho^{1 / 2} \otimes e_{i j}
$$

and the relations

$$
\begin{aligned}
\operatorname{tr}_{1} \omega & =\rho, \\
\operatorname{tr}_{2} \omega & =\left(\mathcal{T} \circ \varphi^{*}\right)(\rho)=\psi^{*}(\rho),
\end{aligned}
$$

hold true.

The dual map $\psi$ can be written as

$$
\psi\left(e_{i j}\right)=\varphi\left(e_{j i}\right)=(\varphi \circ \mathcal{T})\left(e_{i j}\right) \quad \text { or } \quad \psi^{*}=\mathcal{T} \circ \varphi^{*} .
$$

The properties of the composite state $\omega$ can be summarized as follows.

Corollary 1 The composite state $\omega$ is a PPT iff $\varphi$ is $(r, s)$-completely positive.

Corollary 2 The composite state $\omega$ is NPT iff $\varphi$ is $r$-completely positive and $k$-copositive $(k<n)$ provided $\operatorname{rank} \rho=n$. 
The above results imply that the construction of PPT-states is reduced to normalized completely positive and completely copositive maps, while, entangled but not PPT-states are induced by normalized completely positive and $k$-completely copositive maps.

Next we will analyze some examples of normalized completely positive and completely copositive maps.

Example 1 Let us consider the following QCPO:

$$
\pi_{\lambda}=\frac{1-\lambda}{n} I_{n} \otimes I_{n}+\lambda \sum_{i, j=1}^{n} e_{i j} \otimes e_{i j},
$$

where

$$
-\frac{1}{n^{2}-1} \leq \lambda \leq \frac{1}{n+1} .
$$

The above $\pi$ is, up to normalization, the Horodecki state [8], and for $\lambda$ satisfying (2.19) $\pi$ is separable, i.e., $\pi_{\lambda} \in V^{1} \cap V_{1}$.

Let $\psi: M_{n} \rightarrow M_{n}$ be a normalized positive map, then

$$
\sigma_{\lambda}=(\psi \otimes \mathrm{id}) \pi_{\lambda}
$$

is a QCPO which is separable, and the relation

$$
\sum_{i j} \varphi_{\lambda}\left(e_{i j}\right) \otimes e_{i j}=(\psi \otimes \mathrm{id}) \pi_{\lambda}
$$

defines completely positive and completely copositive maps of the form

$$
\varphi_{\lambda}\left(e_{i j}\right)=\frac{1-\lambda}{n} I_{n} \delta_{i j}+\lambda \psi\left(e_{i j}\right),
$$

which is $(1,1)$-completely positive.

Example 2 Let us consider the following QPCO:

$$
\pi_{\gamma}=N_{\gamma}^{-1}\left(n I_{n} \otimes I_{n}+\sum_{i, j=1}^{n} a_{i j} \otimes e_{i j}\right),
$$

where

$$
\begin{aligned}
& a_{i j}=n e_{i j}, \quad i \neq j, \\
& a_{i j}=\left(1-\frac{1}{\gamma^{2}}\right)\left(\gamma^{2} e_{i+1, i+1}-e_{n+i-1, n+i-1}\right)(\bmod n), \\
& N_{\gamma}=n^{2}+\left(1-\frac{1}{\gamma^{2}}\right)\left(\gamma^{2}-1\right), \quad \gamma^{2}>0 .
\end{aligned}
$$

It has been shown in [4] that $\pi_{\gamma} \in V_{2} \cap V^{2}$ but it is not separable. 
The relation

$$
\sum_{i, j=1}^{n} \varphi_{\gamma}\left(e_{i j}\right) \otimes e_{i j}=\pi_{\gamma}
$$

defines a normalized $(2,2)$-completely positive map $\varphi$ which has the form

$$
\varphi_{\gamma}\left(e_{i j}\right)=\frac{1}{N_{\gamma}}\left(n I_{n} \delta_{i j}+a_{i j}\right) .
$$

Example 3 The map $\varphi$ given by (2.28) has the following form

$$
\varphi(a)=\sum_{i, j}^{n} c_{i j} e_{i j} a e_{i j}^{*}+\mu a,
$$

where

$$
\varphi\left(I_{n}\right)=\sum_{i=1}^{n} e_{i i}\left(\sum_{j=1}^{n} c_{i j}+\mu\right) .
$$

Taking into account (2.2) one finds that the relation

$$
\varphi(\mathcal{T}(a))=\mathcal{T}(\varphi(a))
$$

holds.

Theorem 1 The map $\varphi$, as in (2.29), is completely positive iff the following conditions

$$
c_{i j} \geq 0, \quad i \neq j
$$

and

$$
\left[c_{i i} \delta_{i j}+\mu\right] \geq 0
$$

are satisfied.

Proof. The map $\varphi$ can be written in the form

$$
\varphi(a)=\sum_{i, j=1}^{n}\left(\delta_{i j} c_{i i}+\mu\right) e_{i i} a e_{j j}+\sum_{i \neq j} c_{j j} e_{i j} a e_{i j}^{*}
$$

on the other hand the map $\psi$ is completely positive iff it has the form

$$
\psi(a)=\sum_{\alpha, \beta=1}^{n^{2}} \lambda_{\alpha \beta} f_{\alpha} a f_{\beta}^{*},
$$

where

$$
\operatorname{tr}\left(f_{\alpha} f_{\beta}^{*}\right)=\delta_{\alpha \beta}
$$

and

$$
\left[\lambda_{\alpha \beta}\right] \geq 0 .
$$

Taking into account (2.35)-(2.37) and (2.34), one finds conditions (2.33) and (2.34). 
Theorem 2 The map $\varphi$ is completely copositive iff the following conditions

$$
\begin{aligned}
c_{i i}+\mu & \geq 0, \\
c_{i j}+c_{j i} & \geq 2 \mu, \quad i \neq j, \\
c_{i j}+c_{j i} & \geq-2 \mu, \quad i \neq j, \\
c_{i j} c_{j i} & \geq \mu^{2}, \quad i \neq j
\end{aligned}
$$

are satisfied.

Proof. The map $\varphi$ is completely copositive iff the map $\mathcal{T} \varphi$ is completely positive.

Taking into account (2.2) and (2.29) one finds

$$
(\mathcal{T} \circ \varphi)(a)=\sum_{i=1}^{n}\left(c_{i i}+\mu\right) e_{i i} a e_{i i}+\sum_{i \neq j}\left(c_{i j} e_{i j} a e_{i j}^{*}+\mu e_{i j} a e_{i j}\right) .
$$

From (2.42) one finds the condition (2.38). Let us introduce trace orthonormal operators

$$
\begin{aligned}
f_{i j} & =\frac{1}{\sqrt{2}}\left(e_{i j}+e_{j i}\right), \\
g_{i j} & =\frac{-i}{\sqrt{2}}\left(e_{i j}-e_{j i}\right), \quad i<j .
\end{aligned}
$$

Then the equality

$$
\begin{gathered}
\sum_{i \neq j}\left(c_{i j} e_{i j} a e_{i j}^{*}+\mu e_{i j} a e_{i j}\right)=\sum_{k<l}\left\{\left(\frac{1}{2}\left(c_{k l}+c_{l k}\right)+\mu\right) f_{k l} a f_{k l}\right. \\
+\left(\frac{1}{2}\left(c_{k l}+c_{l k}\right)-\mu\right) g_{k l} a g_{k l}-\frac{i}{2}\left(c_{k l}-c_{l k}\right) f_{k l} a g_{k l} \\
\left.+\frac{i}{2}\left(c_{k l}-c_{l k}\right) g_{k l} a f_{k l}\right\}
\end{gathered}
$$

holds true.

Taking into account (2.35) and (2.37) one finds that $\mathcal{T} \circ \varphi$ is completely positive iff the matrices

$$
\Delta_{k l}=\left[\begin{array}{lc}
\frac{1}{2}\left(C_{k l}+C_{l k}\right)+\mu & -\frac{i}{2}\left(C_{k l}-C_{l k}\right) \\
\frac{i}{2}\left(C_{k l}-C_{l k}\right) & \frac{1}{2}\left(C_{k l}+C_{l k}\right)-\mu
\end{array}\right]
$$

are semipositive definite. The conditions (2.39)-(2.41) are equivalent to $\Delta_{k l} \geq 0$.

Corollary 3 The map

$$
\varphi(a)=\sum_{i, j=1}^{n} c_{i j} e_{i j} a e_{i j}^{*}+\mu a
$$


is completely positive and completely copositive, i.e. $\varphi \in P_{n} \cap P^{n}$ iff the following conditions

$$
\begin{aligned}
c_{i j} & >0, \\
c_{i j} c_{j i} & \geq \mu^{2}, \quad i \neq j, \\
c_{i i}+\mu & \geq 0 \\
{\left[c_{i i} \delta_{i j}+\mu\right] } & \geq 0
\end{aligned}
$$

are satisfied.

The $\varphi$ is not normalized, but since $\varphi\left(I_{n}\right)>0$, the map

$$
\psi(a)=\varphi\left(I_{n}\right)^{-1 / 2} \varphi(a) \varphi\left(I_{n}\right)^{-1 / 2}
$$

is normalized, and the state

$$
\sum_{i, j=1}^{n} \rho^{1 / 2} \psi\left(e_{i j}\right) \rho^{1 / 2} \otimes e_{i j}
$$

is a PPT state.

Corollary 4 It follows from Theorems 1 and 2 that choosing $c_{i j}=k, 1 \leq k<$ $n$, and $\mu=-1$ the map

$$
\varphi_{k}(a)=(k-1)\left(\sum_{i=1}^{n} e_{i i} a e_{i i}+\sum_{i<j} f_{i j} a f_{i j}\right)+(k+1) \sum_{i<j} g_{i j} a g_{i j}(2.53)
$$

is completely positive, while the map

$$
\left(\tau \circ \varphi_{k}\right)(a)=k I_{n}(\operatorname{tr} a)-a
$$

is known to be $k$-positive but not $(k+1)$-positive; i.e., $\varphi_{k}$ is completely positive and $k$-copositive, and consequently the state

$$
\frac{1}{k n-1} \sum_{i, j=1}^{n} \rho^{1 / 2} \varphi_{k}\left(e_{i j}\right) \rho^{1 / 2} \otimes e_{i j}
$$

is NPT (by definition) provided $\operatorname{rank} \rho=n$.

Using Theorems 1 and 2 specialized for the case $c_{i i}=c$ and $\mu=-1$.

Corollary 5 The map

$$
\begin{aligned}
\varphi(a) & =\sum_{i=1}^{n}(c-1) e_{i i} a e_{i i}+\sum_{i<j}\left\{\left(\frac{1}{2}\left(c_{i j}+c_{j i}\right)-1\right) f_{i j} a f_{i j}\right. \\
& \left.+\left(\frac{1}{2}\left(c_{i j}+c_{j i}\right)+1\right) g_{i j} a g_{i j}-\frac{i}{2}\left(c_{i j}-c_{j i}\right) f_{i j} a g_{i j}+\frac{i}{2}\left(c_{i j}-c_{j i}\right) g_{i j} a f_{i j}\right\}
\end{aligned}
$$


is completely positive iff $c \geq 1$ and $c_{i j} c_{j i} \geq 1 \quad i \neq j$.

On the other hand

$$
(\tau \circ \varphi)(a)=c \sum_{i=1}^{n-1} h_{i} a h_{i}+\sum_{i \neq j} c_{i j} e_{i j} a e_{i j}^{*}-\frac{n-c}{n} a,
$$

where $h_{1}, h_{2}, \ldots h_{n-1} \in M_{n}, h_{i}=h_{i}^{*}, \operatorname{tr} h_{i}=0, \operatorname{tr} h_{i} h_{j}=\delta_{i j}$ and $\operatorname{tr} h_{i} f_{k l}=$ $\operatorname{tr} h_{i} g_{k l}=0$. Using the result of [9] one finds that $(\tau \circ \varphi)$ is $k$-positive but not $(k+1)$-positive provided the following conditions are satisfied [10]

$$
\begin{gathered}
1 \leq k \leq c<k+1<n, \\
\frac{k}{n-k} \leq \frac{c_{i j}}{n-c}<\frac{k+1}{n-k-1} .
\end{gathered}
$$

In this case the map $\varphi$ generates NPT states.

\section{Acknowledgments}

The work of M. A. and G. M. has been partially supported by a cooperation grant INFN-CICYT. The work of M. A. has also been partially supported by the Spanish MCyT grant FPA2000-1252. A. K. has been supported by the Grant PBZ-MIN-008/P03/2003.

\section{References}

[1] M. Ohya, Lett. Nuovo Cimento 38, 402 (1983).

[2] M. Ohya, IEEE Trans. Information Theory 20, 770 (1983).

[3] N. J. Cerf, C. Adami, arXiv: quant-ph/9610005

[4] K. Życzkowski, I. Bengtson, Open Sys. Information Dyn. 11, 3 (2004).

[5] K. C. Ha, Publ. RIMS Univ. 34, 59 (1998).

[6] M. H. Eom, S. H. Kye, Math. Scan. 86, 130 (2000).

[7] K. C. Ha, S. H. Kye, V.S. Park, Phys. Lett. A 313, 163 (2003).

[8] M. Horodecki, P. Horodecki, R. Horodecki, Phys. Lett. A 223, xxx (1996).

[9] K. C. Ha, Publ. RIMS, Kyoto Univ. 34, xxx (1998).

[10] T. Takasaki, J. Tomiyama, Math. Z. 184, 101 (1983).

[11] P. Arrighi and C. Patricot, On quantum operations as quantum states, Ann. Phys. 311, 26 (2004). 